\title{
Cobalt labelling of single primary auditory neurones: An alternative to HRP
}

\author{
Christine Köppl and Otto Gleich \\ Institut für Zoologie der Technischen Universität München, Garching, F.R.G.
}

(Received 3 August 1987; accepted 31 October 1987)

\begin{abstract}
We have labelled single, primary auditory neurones in three reptile and one bird species. After functional characterization of the neurones, hexamminecobaltic chloride was iontophoretically injected through the recording micropipette. Precipitation of cobalt sulfide followed by silver intensification of the cochlear duct as a whole-mount preparation revealed stained neurones in over $90 \%$ of cases. This method has several advantages over labelling with HRP.
\end{abstract}

Neurone labelling; Auditory nerve; Cobalt labelling; Lizard; Bird; Histological techniques

\section{Introduction}

Cobalt filling has been a standard technique for investigating the branching patterns and projection areas of selected neurones for many years. Originally developed and perfected on insect preparations (Tyrer and Bell, 1974; Bacon and Altman, 1977), it is still used most widely in invertebrates. It has also been successfully used in various vertebrate preparations for the staining of whole nerves or brain pathways (Prior and Fuller, 1973; Iles, 1976; Mason and Lincoln, 1976; Lázár, 1978; Schweitzer and Lowe, 1984; Tóth and Szabó, 1986). However, we know of no reports of singleneurone labelling with cobalt in vertebrates.

The established principles of cobalt labelling are as follows:

Filling of the desired neurone(s) with a soluble cobalt salt. To demonstrate projections of a nerve or connections between brain areas, filling can be accomplished by diffusion, e.g. through the cut end of a nerve (a very popular technique) or by diffusion supported by the application of electri-

Correspondence to: C. Köppl, Institut für Zoologie der Technischen Universität München, Lichtenbergstrasse 4, 8046 Garching, F.R.G. cal current to a bath solution or to a broken cobalt-filled glass electrode. Iontophoretic injection through a microelectrode, both intra- or extracellularly, is used to stain single, physiologically-characterized neurones.

After an appropriate time span (which depends on the temperature and the length of the projections of interest) the cobalt is precipitated as cobalt sulfide. The tissue is then fixed.

The introduction of a third step, the silver intensification, greatly improves the resolution of cobalt staining. This so-called 'physical development' relies on the catalytic action of heavy metals on the reduction of silver nitrate. Thus, cobalt sulfide-containing neuronal profiles act like crystallization nuclei and are greatly enhanced by the deposition of metallic silver. Especially in cases where cobalt concentrations are low, such as in neurones labelled through a microelectrode or in very fine dendritic branches, the stain can only be seen after physical development. Due to its autocatalytic nature, however, the process tends to be difficult to control and somewhat capricious. Thus a number of modifications have been developed since Timm's (1958) original description. The process of physical development is now routinely applied both to wholemounts and to sections (for a review see Gallyas, 1979; Obermayer and Strausfeld, 1980; Tyrer et al., 1980). 


\section{Methods}

The experimental animals used in this study were: Australian bobtail lizards (Tiliqua rugosa), European lizards of the genus Podarcis ( $P$. mura$l i$ and $P$. sicula) and European starlings (Sturnus vulgaris).

We recorded extracellularly either from primary auditory fibres (lizards) or ganglion cell bodies (birds) using glass micropipettes filled with $5 \%$ ( $\approx 190 \mathrm{mM}$ ) hexamminecobaltic chloride. It has been reported by Brogan and Pitman (1981) that hexamminecobaltic chloride is less inclined to block electrodes than cobaltous chloride and that subsequent distribution of the stain is also superior. We were able to use the same electrode types as had previously been found optimal for the respective preparations with conventional $3 \mathrm{M}$ $\mathrm{KCl}$-filling. No difference was seen in the quality of the recordings, although with hexamminecobaltic chloride, the electrodes generally had about 2-4 times the resistance of comparable electrodes with $3 \mathrm{M} \mathrm{KCl}$. We placed successful injections with electrodes pulled from several different glass types (resistances $40-300 \mathrm{M} \Omega$ ). In order to prevent minute particles from blocking the flow of ions, the hexamminecobaltic chloride was filtered through a millipore syringe filter (pore size 0.45 or $0.22 \mu \mathrm{m}$ ) during filling of the electrode.

For injection of the cobalt, positive rectangular pulses ( $50 \mathrm{~ms}$ duration, $10 \mathrm{~s}^{-1}$ ) were applied for 7-45 $\mathrm{min}$ via the bridge circuit of the preamplifier (List LM-1 or WPI M-707A, respectively). The current was usually limited by the electrode and lay between 0.5 and $10 \mathrm{nA}$ (as calculated from the input voltage or current monitor of the preamplifier). The resulting product of current and time could be as low as $3.5 \mathrm{nA} \cdot \mathrm{min}$, the highest used being $210 \mathrm{nA} \cdot \mathrm{min}$. With respect to the current passed, we did not see consistent differences between electrodes made of glass of different wall diameters. The recording was high-pass filtered and continuously monitored during the injection. Sometimes the onset of a blockage, as indicated by vanishing of the recording signal, could be counteracted by one or several brief reversals of pulse polarity. If that did not help, the applied voltage was reduced. In recordings from cell bodies, action potentials and even responses to acous- tic stimuli could frequently be seen during injection. We usually stimulated the neurones during injection with tone pips at the characteristic frequency and at $10-20 \mathrm{~dB}$ above threshold. After injection, we retracted the electrode without penetrating further.

The time span from the end of the iontophoretic injection to the precipitation of cobalt sulfide varied between 1 and $2.5 \mathrm{~h}$ (starlings), 1 and $9.5 \mathrm{~h}$ (European lizards) and 6 and $15 \mathrm{~h}$ (bobtail lizard), the animals being anaesthetized and their body temperature held constant at $30^{\circ} \mathrm{C}$ (lizards) or $40^{\circ} \mathrm{C}$ (birds), respectively. From one stain that had not quite run as far as desired, we estimate the minimum waiting time in lizards to be about $1 \mathrm{~h} / \mathrm{mm}$. The cobalt certainly travels faster in the bird, probably due to the higher temperature, but the minimum time in birds was not determined. We have tried the following precipitating solutions: ammonium sulfide, $0.2-3.5 \%$ (in birds) and $0.5-3.5 \%$ (in lizards) in either $0.9 \%$ $\mathrm{NaCl}$, Ringer's solution or $0.1 \mathrm{M}$ phosphate buffer (pH 7.4), or $1 \%$ sodium sulfide in $0.1 \mathrm{M}$ phosphate buffer. The precipitation was carried out in several different ways: (1) In some lizard preparations, where scala tympani had been opened to record from the auditory nerve, the sulfide solution was applied directly to the exposed basilar papilla and nerve; the fluid was gently withdrawn and replaced about every minute for $5-10 \mathrm{~min}$. This was followed by rinsing with sulfide-free solution with about 5 changes, then by fixation with $4 \%$ formaldehyde in $0.1 \mathrm{M}$ phosphate buffer. (2) In the remaining lizard experiments, the animal was decapitated and the cochlear duct perfused via openings in the oval and round windows. In later experiments, the brainstem was also treated by replacing the fluid in the ventrally-opened braincase. The solutions and time course were the same as above. (3) In bird experiments the whole body was perfused transcardially, first with bird Ringer's solution or $0.9 \% \mathrm{NaCl}$ until the blood had been washed out, then with sulfide solution for 5-6 min and finally with $4 \%$ formaldehyde in $0.1 \mathrm{M}$ phosphate buffer.

The cochlear duct and, in later experiments, the brainstem, were dissected out either immediately afterwards and refrigerated in the fixative (birds), or the whole head was kept in cooled fixative and 
dissected later (lizards). The specimens were carefully freed from surrounding bone and the cochlear ducts opened by removing the tegmentum vasculosum or vestibular membrane, respectively, in order to increase the transparency for later whole-mount observation.

On the next day, or at the most 2 days later, the specimens were washed in distilled water (3-5 changes over a time period of at least $30 \mathrm{~min}$ ). It is important that the tissue is washed thoroughly because residual formaldehyde acts as a reducing agent and can therefore interfere with the following process of physical development (Obermayer and Strausfeld, 1980).

The physical development process used in this study has been described by Bacon and Altman (1977) for wholemount preparations of insect ganglia. The basic incubation medium contains 3 $\mathrm{g}$ gum arabic, $0.17 \mathrm{~g}$ hydroquinone, $10 \mathrm{~g}$ sucrose and $0.8 \mathrm{~g}$ citric acid, dissolved, under stirring, in $100 \mathrm{ml}$ of hot $\left(50-60^{\circ} \mathrm{C}\right)$ distilled water; the final $\mathrm{pH}$ is 2.5-2.6. If desired, this solution can be stored frozen. The second solution required is $1 \%$ aqueous silver nitrate, which is not very stable and should be freshly made up on the same day and shielded from light. The intensification is carried out in the dark at a temperature of $55-60^{\circ} \mathrm{C}$. Clean non-metallic containers must be used for the incubation media; in addition, non-metallic instruments are necessary for transferring and manipulating the specimen. Everything that has contact with the developer solution should either be discarded afterwards or cleaned in concentrated nitric acid before re-use. The specimen is first incubated in warm basic medium in the dark for 1-2 $\mathrm{h}$. Following this, it is transferred to a developer solution ( 1 part $1 \%$ silver nitrate and 9 parts warm basic medium) which has been made up immediately before in a warm container. The progress of development is checked briefly every 5-10 min and stopped when the specimen has turned to a medium brown, by transferring to warm distilled water. Care should be taken not to expose the developing solution to light for very long, nor to allow it to cool down during inspection. The specimen must be transferred to fresh developer at the latest when the solution is turning brown or black due to autocatalytic silver precipitation. In our case, the complete development of a cochlear duct or brainstem took $15-60 \mathrm{~min}$ and the developer was changed every 10-20 min.

The specimen was left in warm distilled water in the dark for another $5 \mathrm{~min}$, then dehydrated in a graded alcohol series and cleared in methyl salicylate for viewing as a wholemount. Specimens were either stored in methyl salicylate or embedded in Spurr's resin and sectioned at $5-10 \mu \mathrm{m}$. Sections were mounted in Canada balsam, because cobalt stains fade within a few weeks under commonly used synthetic mounting media (Peter Bräunig, personal communication).

\section{Results and Discussion}

Our primary aim was to identify the terminal area of the marked auditory neurones within the sensory epithelium of the basilar papilla. Following an initial period of learning the technique and determining optimal parameters, this could be routinely accomplished in the majority of cases. Fig. 1 shows photographs of some typical stains.

In all our experimental animals, the location of terminals was consistent. In different individuals, neurones with the same characteristic frequency projected, with little variation, to the same location in the basilar papilla. Thus we are confident that, at least in cases of single-neurone stains (see below), the neurone from which we recorded immediately before cobalt injection was the labelled one.

In the starling over $90 \%$ (21 of 22) of all trials showed labelled neurones; about $70 \%$ resulted in a single-fibre stain within the basilar papilla, although there could be several less intensely stained cell bodies within the ganglion. In cases where 2 or 3 fibres could be identified all the way to the basilar papilla, the target areas of those fibres always lay close together and did not extend over more than $10 \%$ of the papilla length. In the Australian bobtail lizard, labelling was successful in 7 of 8 trials. In each case, there was only one fibre that could be traced into the basilar papilla. In European lizards, it was more common for more than one neurone to be labelled after a single injection. Although here also, the majority of trials (16 out of $17=94 \%$ ) resulted in stains, about $70 \%$ of those showed between 2 and 6 fibres projecting into the basilar papilla. In addition, in 

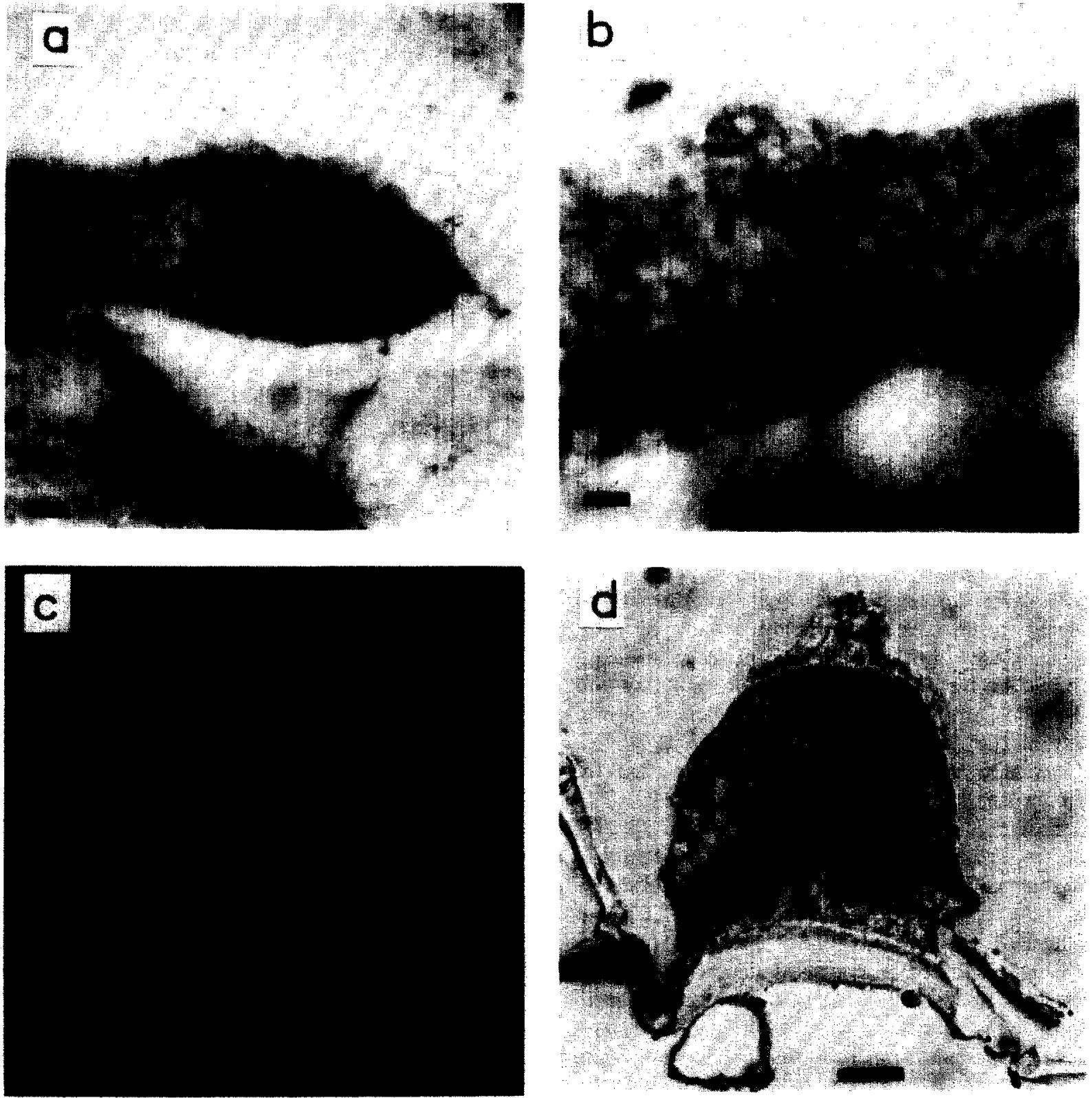

Fig. 1. Some examples of typical cobalt stains. The calibration bar represents $10 \mu \mathrm{m}$ in a, b and d, $100 \mu \mathrm{m}$ in c. (a) A stained nerve fibre contacting a single hair cell in a wholemount preparation of the basilar papilla of a European lizard. Here the hair cells also stained somewhat during the physical development and can be individually distinguished. (b) Clearly defined branching in the terminal area of a single nerve fibre in a wholemount preparation of the basilar papilla of the bobtail lizard. (c) Wholemount of the starling basilar papilla; a single stained fibre enters the papilla and contacts one hair cell. (d) A $5 \mu \mathrm{m}$-thick section of the basilar papilla of a bobtail lizard. The stained (and somewhat swollen) nerve fibre enters from the left. Only part of the terminal branching is visible in this section. 
about half of these multi-label preparations the target areas of the fibres were distributed over more than $15 \%$ of the papilla length, so that they cannot be regarded as specific.

The relatively high rate of non-specific stains in European lizards can be explained by the small size (about 550 fibres) and unusual frequency organization of the auditory nerve, where the characteristic frequency and the site of origin on the basilar papilla of neighbouring fibres can be very different from each other. Thus, uptake of cobalt by neighbouring fibres often results in non-specific staining. Of course, this does not explain why there is a higher tendency for multineurone stains in European lizards as compared to the bobtail lizard and the starling. It should be noted, however, that such variability between species is not restricted to the method of cobalt-labelling. Experience in our laboratory, for example, using single-neurone HRP-labelling has shown that, whereas it very rarely works in the starling cochlea, it works reliably in young chicks under identical conditions (Manley et al., 1987). Even in the chick, however, the pattern of labelling differed between different age groups.

In all species, the intensity of the cobalt stain varied from animal to animal, from medium-brown to black. No consistent correlation could be seen either with the injection parameters (time and current), the different methods of precipitation, or the duration of the physical development. In cases where several neurones were labelled within one specimen, they were often of different intensity, perhaps resulting from different distances to the injection site. Very weak stains could also be due to leakage from the recording electrode, as was seen in one experiment in Podarcis, where the nerve was penetrated with cobalt-filled electrodes about 10 times without placing an electrophoretic injection. Although the electrodes had high resistances (80-500 M $\Omega$ ), indicating a fine tip, the subsequent histology revealed 5 weakly stained cell bodies in the ganglion and 2 just discernible fibres which could, with interruptions, be traced to the point where they entered the papilla. We never found any diffusely stained areas which could have indicated the injection site itself.

In all the animal species, we quite often observed uneven staining in different parts of the same neurone or, occasionally, interruptions where neurones could not be traced all the way through. Weaker staining occurred regularly between the cell body and the basilar papilla, the synaptic terminals usually being intensely stained again. This could be due either to uneven distribution of cobalt within the cell or to uneven precipitation and/or physical development.

Sometimes the unmyelinated part of a fibre and the synaptic terminals appeared swollen. This was most extreme in two cases in the Australian bobtail lizard, where a brown aura around the terminals indicated some leakage of stain. According to Altman and Tyrer (1980), long time spans between the injection of cobalt and its precipitation are the reason for the apparent swelling of fine branches in insect neurones, which they attributed to the loss of cobalt. In our preparations, long waiting times did not necessarily produce swellings, although the extreme cases described above had intervals of more than $12 \mathrm{~h}$ between injection and precipitation.

In European lizards and in the starling, we also tried in some cases to localize the terminal area of the labelled neurone in wholemount preparations of the brainstem. This was never successful in the starling. In all the 3 cases tried, labelled neurones could be traced into the basilar papilla, but the stains faded away beyond the cell body towards the brain. In the lizards, labelled fibres were seen in the trunk of the VIIIth nerve in all cases and in 4 of the 9 animals, neurones could be traced to their terminals within the brain. It is possible that in the other specimens, the stain was too weak to be visible in the rather thick and (due to physical development) dark wholemount.

To summarize, the single-neurone cobalt-labelling technique we describe has a number of advantages over the commonly-used HRP method: It has a very high success rate; the chemicals required are cheap and non-carcinogenic; no special adaptations of the recording electrodes are required and their filling is simple.

The histological preservation of the tissue is, however, not optimal. This, of course, would be a major drawback of the method in those cases where excellent tissue preservation is required. Tyrer et al. (1980) attribute the considerable ultrastructural damage to the treatment with am- 
monium sulfide. It is therefore desirable to minimize the sulfide concentration. In the starling, there was a pronounced tendency for the whole basilar papilla to separate from the basilar membrane when concentrations of ammonium sulfide as high as $3.5 \%$ were used. The precipitation worked reliably with $0.2 \%$ ammonium sulfide and since we did not try anything lower, it may be possible to reduce this even further. Tyrer et al. (1980) recommend using buffered sodium sulfide instead. We have tried this in a few experiments in both lizards and birds, but found that body fluids apparently formed a slimy coagulate upon contact with the sodium sulfide. This, of course, blocks the normal flow of whole-body or ear perfusion and prevents quick and proper fixation. Thus, tissue preservation was actually much worse when sodium sulfide had been used, although stains could still be identified, indicating that the precipitation had worked. Another possibility for improving tissue preservation is to fix the tissue before precipitation (Altman and Tyrer, 1980; Tyrer et al., 1980) or to combine fixation and sulfide treatment (Tyrer and Bell, 1974). We have tried perfusing with fixative for only $3 \mathrm{~min}$ prior to the precipitating solution in 3 starlings. In none of these cases could labelled neurones be subsequently identified.

\section{Acknowledgements}

We thank Peter Bräunig and Geert Runhaar for sharing with us their experience with the cobalt technique in insects. We also thank Geoff Manley, Jim Pickles and Geert Runhaar for commenting on an earlier version of the manuscript. Supported in part by a grant to G. Manley from the Deutsche Forschungsgemeinschaft (SFB 204, "Gehör").

\section{References}

Altman, J.S. and Tyrer, N.M. (1980) Filling selected neurons with cobalt through cut axons. In: N.J. Strausfeld and T.A.
Miller (Eds.), Neuroanatomical Techniques: Insect Nervous System. Springer Verlag, New York, Heidelberg, Berlin.

Bacon, J.P. and Altman, J.S. (1977) A silver intensification method for cobalt-filled neurones in wholemount preparations. Brain Res. 138, 359-363.

Brogan, R.T. and Pitman, R.M. (1981) Axonal regeneration in an identified insect motoneurone. J. Physiol. 319, 34P-35P.

Gallyas, F. (1979) Light insensitive physical developers. Stain Technol. 54, 173-176.

Iles, J.F. (1976) Central terminations of muscle afferents on motoneurones in the cat spinal cord. J. Physiol. 262, 91-117.

Lázár, G. (1978) Application of cobalt-filling technique to show retinal projections in the frog. Neuroscience 3 , 725-736.

Manley, G.A., Brix, J. and Kaiser, A. (1987) Developmental stability of the tonotopic organization of the chick's basilar papilla. Science $237,655-656$.

Mason, C.A. and Lincoln, D.W. (1976) Visualization of the retino-hypothalamic projection on the rat by cobalt precipitation. Cell Tissue Res. 168, 117-131.

Obermayer, M. and Strausfeld, N.J. (1980) Silver-staining cobalt sulfide deposits within neurons of intact ganglia. In: N.J. Strausfeld and T.A. Miller (Eds.), Neuroanatomical Techniques: Insect Nervous System. Springer Verlag, New York, Heidelberg, Berlin.

Prior, D.J. and Fuller, P.M. (1973) The use of a cobalt iontophoresis technique for identification of the mesencephalic trigeminal nucleus. Brain Res. 64, 472--475.

Schweitzer, J. and Lowe, D. (1984) Mesencephalic and diencephalic cobalt-lysine injections in an elasmobranch: evidence for two parallel electrosensory pathways. Neurosci. Lett. $44,317-322$.

Timm, F. (1958) Zur Histochemie der Schwermetalle. Das Sulfid-Silber-Verfahren. Dtsch. Z. Gesamte Gerichtl. Medizin 46, 706-711.

Tóth, P. and Szabó, T. (1986) Simultaneous labelling of two different central nervous system pathways with horseradish peroxidase and cohalt in Gnathonemus petersii and Rana esculenta. Neurosci. Lett. 64, 350-354.

Tyrer, N.M. and Bell, E.M. (1974) The intensification of cobalt-filled neuronc profiles using a modification of Timm's sulphide-silver method. Brain Res. 73, 151-155.

Tyrer. N.M., Shaw, M.K. and Altman, J.S. (1980) Intensification of cobalt-filled neurons in sections (light and electron microscopy). In: N.J. Strausfeld and T.A. Miller (Eds.), Neuroanatomical Techniques: Insect Nervous System. Springer Verlag, New York, Heidelberg, Berlin. 